\title{
La viabilidad de las cooperativas en la globalización: presiones degenerativas y nuevas estrategias de regeneración en las cooperativas multinacionales de Mondragón
}

\author{
Ignacio Bretos FernándeZ \\ Departamento de Dirección y Organización de Empresas, Universidad de Zaragoza \\ ibretos@unizar.es
}

\section{Anjel Errasti Amozarrain}

Instituto GEZKI de Derecho Cooperativo y Economía Social.

Departamento de Economía Financiera II, Universidad del País Vasco (Donostia-San Sebastián) a.errasti@ehu.eus

DOI: $10.1387 /$ reves. 19522

Fecha de entrada: 18/05/2017

Fecha de aceptación: 20/06/2017

\begin{abstract}
Sumario: 1. Introducción. 2. El sistema cooperativo de Mondragón: un retrato actual. 3. Transformación multinacional y presiones degenerativas. 4. Dinámicas de regeneración en las cooperativas multinacionales de Mondragón. 4.1. La revitalización de los valores y prácticas cooperativistas en las empresas cooperativas matrices. 4.2. La extensión del modelo cooperativo en las filiales capitalistas. 5. Conclusiones. 6. Referencias bibliográficas.
\end{abstract}

\section{Resumen:}

Este artículo examina las principales tendencias degenerativas que amenazan a las entidades de Economía Social para desarrollarse en un entorno empresarial cada vez más global y competitivo, y pone en valor la capacidad de estas organizaciones para diseñar e implementar estrategias de regeneración orientadas a revitalizar sus valores y prácticas de carácter social, comunitario y democrático. Para ello, el trabajo se basa en los trabajos empíricos recientes sobre el conocido grupo Mondragón y en la propia investigación cualitativa llevada a cabo por los autores durante los últimos años en diversas cooperativas multinacionales pertenecientes al grupo. Nuestros resultados presentan un desafío importante al determinismo de la 'teoría de la degeneración', la cual muestra signos de hegemonía en el análisis científico de las cooperativas, y contribuyen a una mejor compresión sobre las dinámicas de regeneración en las cooperativas en el con- 
texto de la globalización, un ámbito donde la literatura previa es extremadamente limitada. También se exponen las implicaciones de esta investigación para la teoría organizacional de las cooperativas.

\title{
Palabras clave:
}

Degeneración, Regeneración, Economía Social, Cooperativas, Mondragón.

\begin{abstract}
:
This paper examines the main degenerative tendencies that threaten Social Economy firms to develop in an increasingly global and competitive business environment, and highlights the ability of these organizations to design and implement regeneration strategies aimed at revitalizing their values and practices of social, communitarian and democratic nature. To this end, the article draws on recent empirical studies on the well-known Mondragon cooperative group as well as on the qualitative research carried out by the authors in the last years in a number of multinational cooperatives belonging to the group. Our findings pose an important challenge to the determinism of the 'degeneration thesis', which shows signs of hegemony in the scientific analysis of cooperatives, and contribute to a better understanding of the regeneration dynamics in cooperatives in the context of globalization, a field in which the previous literature is extremely limited. The implications of this research for the organizational theory of cooperatives are also discussed.
\end{abstract}

\section{Keywords:}

Degeneration, Regeneration, Social Economy, Cooperatives, Mondragon.

\section{Claves Econlit:}

A130, F23, J54, M54.

\section{Introducción}

La viabilidad de las cooperativas en un entorno capitalista ha sido un tema central de debate avanzado por el Marxismo desde finales del siglo xix. Sobre la discusión del poder emancipador de las cooperativas en la esfera de la producción, Marx argumentó que estas organizaciones pueden constituir una fuerza transformadora en tanto que reflejan las posibilidades estructurales de la producción social democrática. Sin embargo, también señaló las contradicciones a las que están sujetas estas organizaciones en tanto que deben florecer y desenvolverse en un sistema preeminentemente capitalista (Marx, 1967). La tradición marxista desplegó esta tesis en térmi- 
nos más pesimistas, sugiriendo que las cooperativas únicamente reproducen los defectos del modelo capitalista (Luxemburg, 1900; Mandel, 1975). De manera similar, socialistas fabianos como Sidney y Beatrice Webb (Webb y Webb, 1914, 1920) sostenían que las cooperativas no son opciones viables a largo plazo, ya que tienden a colapsar como 'democracias de productores' y virar hacia 'asociaciones de capitalistas' en un proceso mediante el cual la participación de los trabajadores disminuye sensiblemente, el poder de decisión y control queda en manos de una oligarquía, la obtención de beneficios se convierte en un objetivo primordial, y el colectivo de socios es reemplazado paulatinamente a través de la contratación de trabajadores asalariados.

Estos trabajos dieron lugar al posterior desarrollo, fundamentalmente durante las décadas de 1970 y 1980, de la denominada 'teoría de la degeneración' (entre otros, Meister, 1974, 1984; Ben-ner, 1984; Russell, 1984; Miyazaki, 1984), la cual sugiere que las cooperativas están inexorablemente destinadas a fracasar comercialmente o a degenerar en formas empresariales convencionales bajo modelos organizativos y prioridades similares a las de la empresa capitalista. No obstante, un grupo más reducido de trabajos académicos plantearon una alternativa a esta visión tan determinista y negativa sobre el ciclo de vida de las cooperativas, desarrollando la denominada 'teoría de la regeneración' (Batstone, 1983; Rosner, 1984; Stryjan, 1994; Cornforth, 1995), la cual sostiene que las cooperativas son capaces de mantener su naturaleza original a largo plazo, y que la degeneración puede ser una etapa temporal del ciclo de vida cooperativo seguida por la dinamización de procesos de regeneración capaces de restaurar el funcionamiento democrático, participativo y social de estas organizaciones.

En las dos últimas décadas marcadas por la intensificación del proceso de globalización neoliberal (Harvey, 2005), ha emergido un renovado debate en torno a la viabilidad de las cooperativas ante las nuevas condiciones impuestas por la globalización y su capacidad para retener sus prácticas y valores cooperativistas a la vez que mantienen su competitividad en los mercados y su eficiencia en términos económico-empresariales (Atzeni, 2012; Parker et al., 2014; Bretos y Marcuello, 2017). Como destacaban recientemente Storey et al. (2014), históricamente, la degeneración ha sido el prisma que ha dominado el análisis de las cooperativas y sigue mostrando signos de hegemonía científica (véase también Cornforth, 1995 para una extensa crítica de la literatura sobre la degeneración cooperativa). En este sentido, diversos trabajos han incidido en las tendencias degenerativas experimentadas, en términos democráticos y participativos, por cooperativas que operan en mercados de gran dinamismo (Varman y Chakrabarti, 2004; Cathcart, 2013, 2014; Paranque y Willmott 2014). Por el contrario, otros trabajos se han centrado en las posibilidades de las cooperativas para 
revitalizar la participación de los trabajadores en la empresa y hacer frente a las presiones gerencialistas y oligárquicas que pueden darse dentro de estas organizaciones (Hernandez, 2006; Ng y Ng, 2009; Storey et al., 2014; Jaumier, 2017; Narvaiza et al., 2017). No obstante, estos estudios han estado generalmente limitados al análisis de pequeñas y medianas cooperativas que desarrollan su actividad exclusivamente a nivel doméstico.

Por tanto, si bien esta literatura previa ha proporcionado contribuciones esenciales, es evidente que nuestro conocimiento sobre las dinámicas degenerativas y regenerativas que tienen lugar en grandes cooperativas multinacionales es extremadamente limitado. Esta investigación es esencial teniendo en cuenta que las cooperativas, al igual que otras empresas de reducida dimensión tradicionalmente locales, están viéndose obligadas a desarrollar estrategias de internacionalización en aras de sobrevivir en sectores cada vez más competitivos y globalizados (McMurtry y Reed, 2009; Bretos y Marcuello, 2017). El conocido grupo cooperativo Mondragón conforma un fructífero terreno para estudiar estas cuestiones, en tanto que muchas de sus cooperativas industriales están organizadas como empresas multinacionales. Debido al crecimiento internacional y la competencia global, estas cooperativas multinacionales han sufrido un proceso de degeneración basado en el deterioro de los valores sociales y prácticas cooperativistas. Sin embargo, en los últimos años, se han promovido diversas estrategias de regeneración con el objetivo de frenar y revertir dicho proceso degenerativo. En concreto, nuestro trabajo explora, por un lado, las tendencias degenerativas experimentadas por las cooperativas multinacionales de Mondragón a través del deterioro de la participación de los trabajadores en favor del mayor control gerencial, la redefinición de los valores cooperativos de acuerdo con los intereses de eficiencia económica y productividad, y el establecimiento de filiales capitalistas donde los trabajadores son asalariados. Por otro lado, examinamos las distintas estrategias regenerativas puestas en marcha en estas cooperativas multinacionales. Si bien se realiza un breve repaso a las experiencias de regeneración dirigidas a revitalizar la participación de los socios trabajadores en las cooperativas matrices, el estudio se centra principalmente en analizar las iniciativas de regeneración orientadas a exportar el modelo cooperativo a las filiales capitalistas dada la vital importancia de este tipo de experiencias.

Para ello, este estudio se basa en trabajos empíricos recientes sobre el grupo Mondragón, así como en la propia investigación cualitativa llevada a cabo por los autores durante los últimos años en algunas de las cooperativas multinacionales más importantes del grupo, como Fagor Ederlan, Maier y Fagor Electrodomésticos. Dicha investigación cualitativa utiliza datos primarios y secundarios. Por un lado, los datos primarios se refieren a la realización de multitud de entrevistas en profundidad con altos car- 
gos de la Corporación Mondragón y diferentes actores organizacionales tanto de las cooperativas matrices como de las filiales domésticas y extranjeras (por ejemplo, directivos, expatriados, socios, representantes sindicales, trabajadores asalariados y temporales, y representantes de los órganos de gobierno de las cooperativas). Por otro lado, los datos secundarios se obtuvieron a partir de diversa documentación interna proporcionada por Mondragón y las cooperativas (informes anuales, memorias de sostenibilidad y responsabilidad social, planes estratégicos, estatutos sociales, etc.) y de la información disponible públicamente, por ejemplo, en notas de prensa, revistas corporativas y documentos audiovisuales.

Tras esta introducción, el siguiente apartado muestra una fotografía actual del grupo Mondragón, incidiendo en la dimensión multinacional de sus cooperativas industriales. El tercer apartado se centra en analizar las distintas tendencias degenerativas experimentadas por las cooperativas multinacionales de Mondragón, mientras que el cuarto apartado explora las estrategias de regeneración más relevantes puestas en marcha. Finalmente, el último apartado recoge las principales conclusiones del estudio y extrae algunas implicaciones esenciales para el desarrollo de la teoría organizacional de las cooperativas.

\section{El sistema cooperativo de Mondragón: un retrato actual}

Un aspecto clave sobre el que se erige el sistema regional de gobernanza del País Vasco es el 'asociacionismo' (Cooke, Uranga, y Etxebarria, 1997), sostenido en una larga tradición de activismo obrero, democracia organizacional y participación en el ámbito laboral y comunitario (Caro Baroja, 1974). Hoy en día, este patrón histórico se refleja en la notable presencia de empresas participadas en la región vasca (Bretos y Morandeira, 2016). La Experiencia Cooperativa de Mondragón, originada hace más de medio siglo en Arrasate-Mondragón (Gipuzkoa), es probablemente la mejor representación de este entorno institucional (Whyte y Whyte, 1991; Kasmir, 1996; Cheney, 2002). Es significativo señalar que, mientras en el resto de España la tasa de paro alcanzó el 25\% en 2012, en el País Vasco se mantuvo en el $12 \%$ y en los tres valles donde se concentran las cooperativas de Mondragón no superó el 5\% (Webb y Cheney, 2014).

Desde sus inicios, la Experiencia Cooperativa de Mondragón fue una experiencia anclada en las necesidades del entorno local, creándose en 1943 la actual Universidad de Mondragón, en 1956 la cooperativa industrial Ulgor (posteriormente Fagor Electrodomésticos), y en 1959 la entidad de crédito cooperativo Laboral Kutxa y el sistema de protección social Lagun Aro. Estas cuatro ramas enarbolaron el desarrollo de la comunidad, 
impulsaron cientos de cooperativas, y consolidaron la hoy denominada Corporación Mondragón (Mondragón, 2015). Inspiradas por la doctrina social católica del padre Arizmendiarrieta, estas cooperativas comenzaron como pequeñas organizaciones democráticas, con profundas raíces en el territorio y un poderoso sentido de comunidad, en torno al concepto $h u$ man community work, basado en la noción de que todos los trabajadores eran socios y copropietarios de la empresa (Molina y Miguez, 2008). Un objetivo primordial, todavía hoy vigente, fue promover el desarrollo humano de las personas en el trabajo y alentar la creación de nuevas cooperativas y puestos de trabajo en aras de impulsar el desarrollo socioeconómico en la región (Mondragón, 2015). Históricamente, las organizaciones agrupadas en la Corporación Mondragón se han acogido a los siguientes principios:

1. Libre adhesión.

2. Organización democrática.

3. Soberanía del trabajo.

4. Carácter instrumental y subordinado del capital.

5. Participación en la gestión.

6. Solidaridad retributiva.

7. Intercooperación.

8. Transformación social.

9. Carácter universal.

10. Educación.

Sin embargo, la Experiencia Cooperativa de Mondragón ha experimentado una transformación extraordinaria durante las últimas décadas. Las presiones competitivas y económicas de la globalización han obligado a muchas de sus cooperativas industriales a perseguir una intensa estrategia de crecimiento, primero en el mercado doméstico, y desde principios de la década de 1990 también en los mercados internacionales. Actualmente, la Corporación Mondragón es el primer grupo empresarial del País Vasco y el décimo en el ránking de las empresas españolas. El grupo está configurado como una federación que emplea 74.335 personas en 261 organizaciones (de las cuales 101 son cooperativas) distribuidas en las áreas de industria, finanzas, conocimiento y distribución. El área de Finanzas incluye la actividad de banca, previsión social y seguros. El área de Industria agrupa 12 divisiones industriales dedicadas a la producción de bienes y servicios. El área de Distribución aglutina a las cooperativas y negocios de distribución comercial y la actividad agroalimentaria. El área de Conocimiento incluye la red de centros tecnológicos y unidades de investigación y desarrollo (I+D), Mondragon Unibertsitatea, y varios centros de formación profesional y de enseñanza. Como se puede ver en la Figura 1, el corazón industrial de 
Mondragón está compuesto por unas 25 cooperativas multinacionales que controlan cerca de 130 plantas productivas en el extranjero. Estas filiales emplean 11.796 personas, lo que representa cerca del $40 \%$ del empleo total de la división industrial de Mondragón. En cuanto a las ventas, más de un 70\% corresponden a ventas internacionales (Mondragón, 2016).

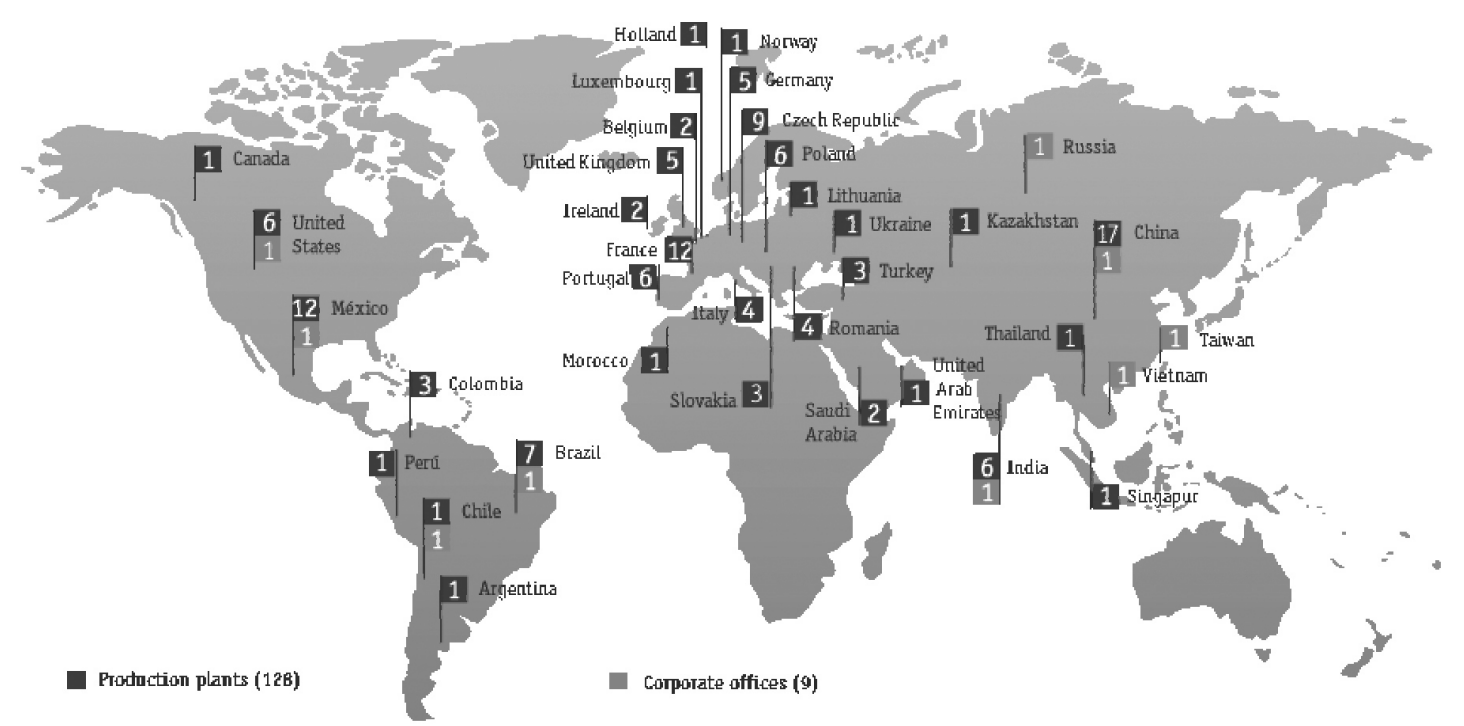

Fuente: Mondragón (2016).

Figura 1

Presencia internacional de la Corporación Mondragón

Esta transformación se refleja en la redefinición de la Experiencia Cooperativa de Mondragón bajo el concepto humanity at work, cuya nueva misión 'combina los objetivos centrales de una organización empresarial que compite en los mercados internacionales con la utilización de métodos democráticos en su organización societaria, la creación de empleo, la promoción humana y profesional de sus trabajadores y el compromiso de desarrollo con su entorno social' (Mondragón, 2015: 21). A pesar de ello, los valores y prácticas cooperativas siguen guiando el funcionamiento de las cooperativas matrices de Mondragón en el País Vasco. Como propietarios, los socios trabajadores participan en el reparto de beneficios y están involucrados en la toma de decisiones de diversas formas. Por un lado, participan en la Asamblea General bajo la regla «una persona/un voto» y pueden ser elegidos como miembros del Consejo Rector y del Consejo Social (Cheney, 2005) (la estructura de gobierno de una cooperativa se representa en la Figura 2). 


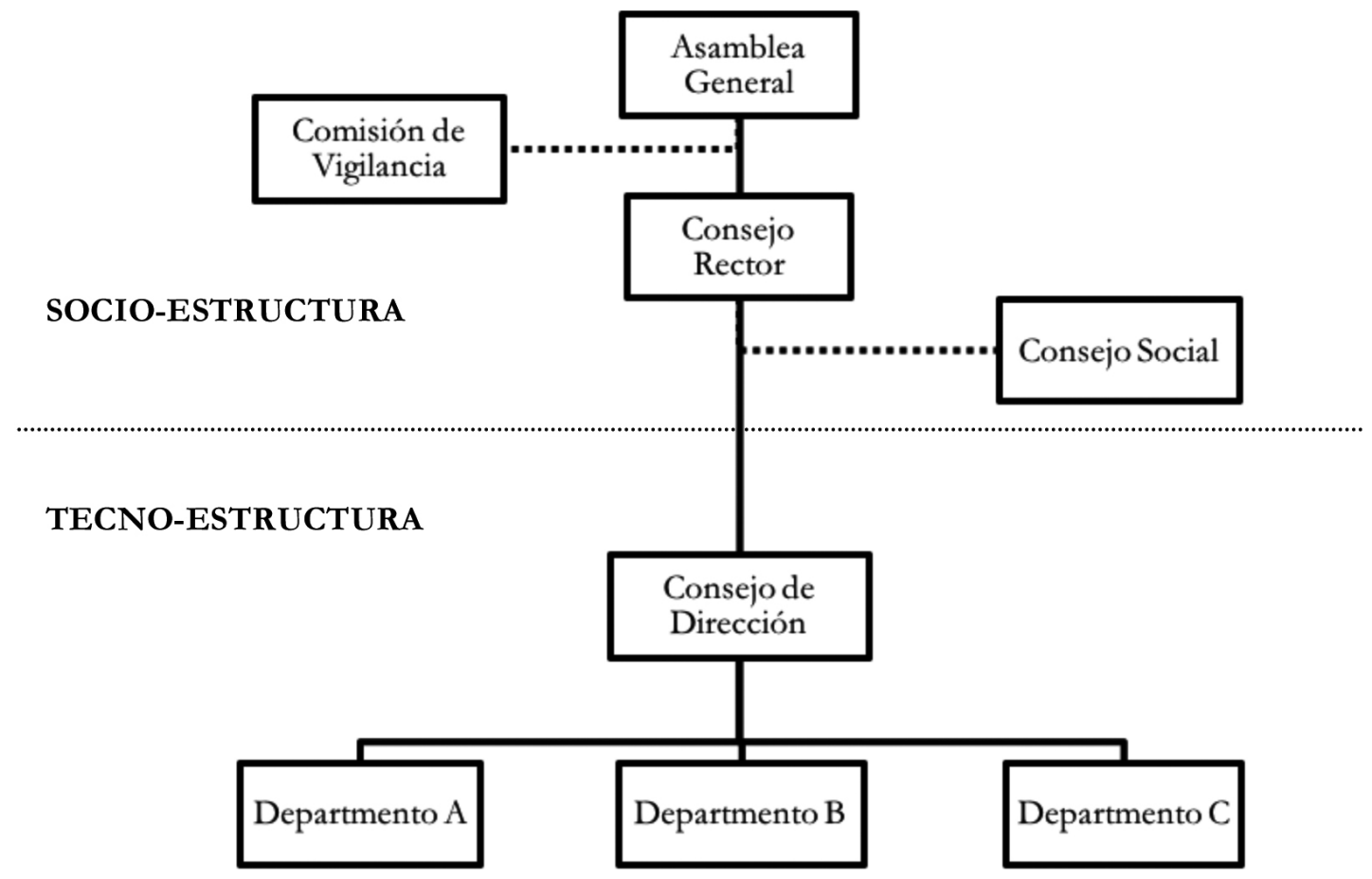

Fuente: adaptado de Altuna et al. (2013) y Whyte y Whyte (1991).

Figura 2

Estructura de gobierno de una cooperativa de Mondragón

La Asamblea General es el órgano supremo de la cooperativa que expresa la voluntad social expresada por todos los socios. Está compuesto por todos los socios de la cooperativa y se reúne al menos una vez al año. Este órgano aprueba los planes estratégicos de la cooperativa y nombra al Consejo Rector, el Consejo Social y la Comisión de Vigilancia. El Consejo Rector es el órgano de representación y gobierno de la cooperativa, y sus miembros son elegidos en Asamblea General. Este órgano es responsable de gobernar y representar a la compañía, y sus decisiones están subordinadas a las políticas y estrategias fijadas por la Asamblea General. Supervisa la administración, nombra al gerente y supervisa su desempeño. La Comisión de Vigilancia cuyo propósito es dictaminar sobre el correcto cumplimiento de los aspectos contables y de aquellos otros que requieran su consideración. El Consejo Social cumple un papel similar al sindicato. Es un órgano consultivo que representa a los socios ante las instancias internas de la cooperativa. Tiene un papel consultivo y de comunicación social, y actúa como canal entre la gerencia y los trabajadores. Los miembros son elegidos por áreas de trabajo y son ratificados por la Asamblea General. Sus funciones son el asesoramiento laboral, la información, la negociación y el 
control social. Por último, el Consejo de Dirección es el órgano ejecutivo que gestiona la cooperativa. Está constituido por los miembros directivos y el gerente, que es nombrado por el Consejo Rector y puede ser restituido por los socios (Altuna et al. 2013; Azkarraga et al., 2012; Whyte y Whyte, 1991).

Por otro lado, los trabajadores participan en el área de trabajo por medio de distintos mecanismos, como las reuniones conjuntas entre trabajadores y dirección (Arando et al., 2015). Igualmente, la promoción interna y la estabilidad laboral también son primordiales en Mondragón (Heras, 2014). El despido de socios es extremadamente inusual y, cuando una planta ocasionalmente cierra, los socios son reubicados en otras cooperativas del grupo. Asimismo, las diferencias salariales en las cooperativas del grupo son notablemente reducidas, aunque han aumentado desde la escala original 1:3 a la actual 1:7 en algunos casos. Por último, cabe señalar que los socios reciben formación tanto en aspectos técnicos y de negocio como en la cultura y valores de la Experiencia Cooperativa de Mondragón (Basterretxea y Albizu, 2011).

\section{Transformación multinacional y presiones degenerativas}

Aunque las cooperativas de Mondragón han estado sujetas desde sus inicios a demandas contradictorias entre las lógicas institucionales democráticas y de mercado, los cambios provocados por la creciente globalización y competencia en los mercados desde la década de 1980 han intensificado estas tensiones (Taylor, 1994). La internacionalización es una clara consecuencia. Algunas cooperativas industriales de Mondragón se han visto obligadas a crecer internacionalmente desde principios de los 90 para seguir siendo competitivas y salvaguardar los empleos de los socios trabajadores en las plantas vascas, ante la entrada de diversas multinacionales en el mercado doméstico como consecuencia de la incorporación de España en la Unión Europea en 1986.

La internacionalización y la competencia global han influido de varias maneras en la transformación de los valores y prácticas originales de estas cooperativas (véase también Bretos y Errasti, 2017; Bretos et al., 2017). Nuestra investigación identificó diversas dinámicas que afectan fundamentalmente a la gobernanza y a la naturaleza y alcance de la participación de los trabajadores en las cooperativas. En primer lugar, el mayor tamaño organizacional de las cooperativas, así como la mayor complejidad de las decisiones estratégicas que hay tomar al estar inmersos en dinámicas empresariales cambiantes y globales, han sido factores clave que han afectado a la participación de las personas. Diversos socios entrevistados reconocieron 
una cierta ritualización de las Asambleas Generales y otros espacios democráticos, señalando que se habían convertido en espacios simbólicos más que en estructuras donde la gente realmente participaba; aspectos resaltados también en otros trabajos previos sobre cooperativas del grupo Mondragón (Greenwood y Gonzalez, 1991; Kasmir, 1996).

Asimismo, la intensificación de los requisitos de eficiencia económica, derivada de la competencia global y el crecimiento internacional, ha favorecido que la auto-gestión y participación se hayan visto desplazados en favor de tendencias oligárquicas y gerencialistas (Heras, 2014). Esta transformación ha sido impulsada por el mayor poder de control depositado en directivos que, a menudo, están más comprometidos con la eficiencia que con la cultura cooperativa, así como por un discurso gerencialista centrado en la competitividad que privilegia los intereses gerenciales de rentabilidad y crecimiento (Taylor, 1994; Heras y Basterretxea, 2016).

Estos factores se reflejan en el hecho de que las cooperativas multinacionales de Mondragón han importado regímenes dominantes de gestión y productividad (Cheney, 2005). Un ejemplo es la introducción de los círculos de calidad y otras técnicas articuladas bajo el paradigma de la Gestión de la Calidad Total, aspecto central alrededor del cual gira la cultura de las cooperativas de Mondragón (Heras, 2014). Por ejemplo, a principios de la década de los 2000, muchas cooperativas introdujeron el sistema de las 'mini-compañías' con el objetivo de fomentar la eficiencia y la productividad a través de la mayor motivación de los trabajadores. Las mini-compañías son, en pocas palabras, una forma de estructurar la organización para que cada una de sus unidades funcione como una pequeña empresa autónoma en la cual los trabajadores toman decisiones y resuelven problemas relacionados con el área de trabajo en el mismo lugar donde tienen lugar. Como se observó en nuestra investigación, estos modelos de gestión han inculcado una cultura débil y superflua de autogestión, que promueve formas de participación controladas gerencialmente, limitadas a niveles bajos de decisión en el área de trabajo, y evaluadas en términos de motivación de los empleados y compromiso con los objetivos productivos marcados desde arriba (véase también Cheney, 2002; Heras, 2014; Bretos y Errasti, 2017; Bretos et al., 2017).

Por otra parte, el propio modelo de internacionalización seguido por las cooperativas de Mondragón constituye una contradicción. Por un lado, la estrategia de internacionalización se ha basado en la denominada 'multilocalización' (Luzarraga, 2008), esto es, una estrategia expansionista ya que la nueva actividad abierta en el exterior no implica el cierre de ninguna actividad preexistente en el mercado doméstico. No obstante, por otro lado, el crecimiento tanto a nivel doméstico como internacional se ha basado en el establecimiento de filiales no cooperativas (sociedades capitalistas) donde 
sus trabajadores son asalariados y, por tanto, no disfrutan de los mismos derechos que los socios cooperativistas de las matrices, es decir, no participan en la propiedad, reparto de beneficios y gestión de sus empresas (Bretos y Errasti, 2017; Bretos et al., 2017). De hecho, aunque las cooperativas matrices han mantenido una elevada proporción de socios cooperativistas sobre empleados asalariados (alrededor del 80\% empleo está conformado por el colectivo de socios), contando también el empleo de las filiales, esta proporción baja al 30\%. En este sentido, entre 1991 y 2007, la proporción de empleo cooperativo en el conjunto del Grupo Mondragón disminuyó del 86\% al 29,5\% (Storey et al., 2014).

En términos generales, la multilocalización ha proporcionado resultados extraordinarios a las cooperativas de Mondragón, favoreciendo la creación de empleo tanto en el País Vasco como en el exterior, y dotándoles de flexibilidad para afrontar la recesión económica en mejores condiciones (Elortza et al., 2012; Luzarraga e Irizar, 2012). Por ejemplo, el empleo aumentó en el Grupo Fagor Ederlan de 1.300 trabajadores en 1999 a 3.700 en 2015. En cambio, este patrón no fue tan evidente con la caída de Fagor Electrodomésticos, hecho de gran impacto económico, social y simbólico por ser el buque insignia del grupo Mondragón. La multilocalización brindó a Fagor resultados extraordinarios durante años y ayudó a capear varios ańos de crisis económica al contrarrestar la caída de la demanda doméstica de electrodomésticos y los altos costos laborales en España. Sin embargo, si en 2007 el grupo contaba con 11.000 trabajadores, antes de su colapso en 2013 sólo quedaban 5.500 trabajadores en el grupo (1.900 de ellos en el País Vasco). Cabe destacar que la caída de esta cooperativa se debió fundamentalmente a las condiciones del mercado, aunque ello no le exime de diversos problemas relacionados con la gobernanza y la toma de decisiones como los analizados anteriormente (para un análisis detallado sobre la caída de Fagor, véase Errasti, Bretos y Etxezarreta, 2016; Errasti, Bretos, y Nunez, 2017).

Los mecanismos internos del sistema Mondragón han sido clave para hacer frente a este escenario. Hoy en día, la inmensa mayoría de los socios cooperativistas afectados por el cierre de Fagor en el País Vasco han encontrado una solución, principalmente mediante su reubicación en otras cooperativas del grupo. En cambio, los miles de trabajadores asalariados (no socios) de la cooperativa y sus filiales han quedado excluidos de los mecanismos de solidaridad de Mondragón. Ello pone en relieve las contradicciones de estas cooperativas multinacionales no sólo en lo relativo a las carencias democráticas y participativas en las filiales capitalistas sino también en términos de la naturaleza del empleo y las condiciones socio-laborales en dichas filiales (Kasmir, 2016). Por ejemplo, existe evidencia que señala que las condiciones laborales en las filiales chinas de Mondragón no difie- 
ren significativamente de las existentes en otras filiales de multinacionales capitalistas del entorno (Errasti, 2015). Entre otras cuestiones, ello se refleja en las diferencias salariales entre los trabajadores chinos, quienes ganan 1,5 euros/hora, y los socios cooperativistas vascos, con un salario de alrededor de 21 euros/hora. Esta problemática no es exclusiva al caso de China. En países como Polonia o Francia se han producido fuertes protestas sindicales contra las condiciones laborales existentes en filiales de Mondragón (Errasti et al., 2016).

\section{Dinámicas de regeneración en las cooperativas multinacionales de Mondragón}

Tras décadas de intenso crecimiento que han alejado a algunas cooperativas de Mondragón de su naturaleza social y cooperativa en favor de las prerrogativas gerenciales y la orientación al mercado, el grupo vive inmerso desde mediados de los años 2000 en un proceso de reflexión y debate orientado a revitalizar aspectos esenciales del modelo cooperativo como la transformación social y comunitaria, la participación de los trabajadores, la gobernanza democrática, la intercooperación, y la formación y educación cooperativa (Azkarraga et al., 2012). Esta reflexión ha resultado en la implementación de estrategias de regeneración en diversas cooperativas del grupo. De manera general, existen iniciativas de regeneración orientadas a la revitalización de las prácticas y valores originales en las cooperativas matrices, por un lado, y a la extensión del modelo cooperativo a las filiales capitalistas, por el otro.

\subsection{La revitalización de los valores y prácticas cooperativistas en las empresas cooperativas matrices}

Varias cooperativas industriales del grupo Mondragón, de gran tamaño y naturaleza multinacional, están tratando de impulsar, particularmente desde el año 2005, proyectos de recuperación y revitalización de los valores y prácticas cooperativistas originarias (Azkarraga et al., 2012; Webb y Cheney, 2014).

Mientras que en la década de los 90 la educación en los aspectos filosóficos, sociales y prácticos del cooperativismo pasó a un segundo plano en favor de la capacitación técnica (Cheney, 2002), en los últimos años existen diversos proyectos - diseñados e impulsados por el Instituto LANKI de estudios cooperativos de la Universidad de Mondragón y por el centro de formación cooperativa y de gestión Otalora - para reintroducir, renovar e ins- 
titucionalizar la formación y educación cooperativista en las cooperativas del grupo (Webb y Cheney, 2014). En términos generales, se está tratando no sólo de fortalecer las competencias directivas y facilitar el desarrollo profesional de los directivos, sino también aspectos como el desarrollo cultural (centrado en fomentar una dirección empresarial acorde a una cultura organizacional más cooperativa y constructiva), la educación cooperativa (dirigida a proporcionar capacitación a los miembros de los órganos sociales para que desempeñen su rol de manera competente y a promover el sentimiento de pertenencia de los socios trabajadores a la cultura y valores cooperativas), y habilidades sociales como el liderazgo cooperativo y el trabajo en equipo (Azkarraga et al., 2012; Basterretxea y Albizu, 2011).

Esta revitalización en la educación y formación cooperativa sirve también como apoyo a los proyectos que se están desarrollando para reforzar la participación tanto en los órganos sociales como en el área de trabajo. Ello se está llevando a cabo fundamentalmente mediante la reconfiguración y profundización de la comunicación en las cooperativas. Por un lado, desde hace ańos, se vienen promoviendo en diversas cooperativas reuniones preparatorias previas a las asambleas generales, realizadas en grupos pequeños de unos 30-40 socios, con el objetivo de facilitar la transmisión de información y fomentar la participación en estos espacios. Las charlas informativas que se han mantenido tradicionalmente para informar a los trabajadores sobre cuestiones clave de la cooperativa se están dinamizando con el objetivo de promover una mayor participación, diálogo y reflexión. Asimismo, la estructura del Consejo Social ha sido objeto fundamental de iniciativas transformadoras e innovadoras. En los últimos años, diversas cooperativas han creado los denominados "consejillos», esto es, reuniones periódicas que tienen por objeto facilitar la comunicación entre los trabajadores de base y los representantes del Consejo Social. Ante la saturación del Consejo Social derivada de su utilización por parte de los trabajadores como medio para expresar sus quejas, problema habitual en las cooperativas de Mondragón, Fagor Ederlan ha creado las denominadas «reuniones sociales de planta». Mensualmente, una persona de la Comisión Permanente del Consejo Central, el responsable de planta, una persona del equipo de Gestión Social y los vocales sociales de la planta, se reúnen para compartir la información sobre la gestión de la planta, resolver los problemas sociales de su ámbito y proponer temas para trasladar al Consejo Social. Asimismo, también se han creado los «consejos sociales de negocio», que reúnen a una persona del Consejo Rector, el Director de Línea de Producto, un miembro de Gestión Social y los vocales sociales adscritos al negocio. En estos espacios se comparte información relativa a la gestión, se discuten temas generales de la cooperativa y se abordan temas sociales que escapan del ámbito de las reuniones de planta. 
Otras cooperativas industriales particularmente dinámicas e innovadoras, como Fagor Arrasate, también están experimentando internamente con nuevas formas de participación (Webb y Cheney, 2014). Esta cooperativa ha propiciado dinámicas más participativas mediante un tratamiento más cualitativo de la información y la apertura de espacios para la deliberación y la participación más activa de las personas. Por ejemplo, el rol del consejero se ha redefinido para no sólo informar sino también dinamizar la participación en los consejillos. Igualmente, los consejillos se han reconfigurado para fomentar el rol asesor del Consejo Social.

\subsection{La extensión del modelo cooperativo en las filiales capitalistas}

Más allá de estos aspectos, que atañen fundamentalmente a la revitalización de la participación y gobernanza democrática en las cooperativas matrices vascas, un aspecto particularmente relevante y novedoso consiste en la aprobación en el año 2003 de la denominada 'estrategia de expansión social' por parte del Congreso Cooperativo de Mondragón. Dicha estrategia se refiere a la difusión de los valores cooperativos en las filiales capitalistas mediante el desarrollo de mecanismos de participación para los trabajadores similares a los existentes en las cooperativas (Irizar, 2005). Este Congreso acordó objetivos cruciales como fomentar una mayor transparencia en la toma de decisiones en las filiales, implementar el mismo modelo de gestión participativa que en las cooperativas, avanzar hasta al menos un $30 \%$ de la propiedad en manos de los trabajadores, y dedicar entre el $1 \%$ y $5 \%$ de los beneficios al desarrollo de los territorios donde están localizadas las filiales (Flecha y Ngai, 2014). Posteriormente, el Modelo de Gestión Corporativo de Mondragón ${ }^{1}$ incluyó tres aspectos esenciales a profundizar en las cooperativas: la Autogestión, la Comunicación y el Desarrollo Corporativo (Mondragón, 2013).

Los dos primeros aspectos (Autogestión y Comunicación) abarcan tanto a las cooperativas como a sus filiales, mientras que el tercer aspecto (Desarrollo Corporativo) está diseñado fundamentalmente para las segundas. Respecto a la Autogestión en las cooperativas y filiales, la Corporación incide en diseñar estructuras organizativas horizontales que faciliten la par-

${ }^{1}$ El Modelo de Gestión Corporativo es la herramienta general creada para homogenizar la gestión de todas las cooperativas del grupo Mondragón y sus filiales. El Modelo de Gestión Corporativo no es un plan de acción detallado ni implica su obligado cumplimiento por parte de las cooperativas del grupo, sino que proporciona unas pautas generales que cada cooperativa adapta a su contexto particular, dirigidas a lograr una gestión empresarial que sea a la vez eficiente y consistente con la cultura corporativa de Mondragón (Mondragón, 2013). 
ticipación y el trabajo en equipo y posibiliten la definición colectiva de objetivos y la asunción de responsabilidades. La Comunicación se centra en establecer políticas de transparencia que fomenten las relaciones interpersonales y la fluidez de la información en toda la organización. Finalmente, el Desarrollo Corporativo se centra en exportar el modelo cooperativo a las capitalistas mediante la introducción del modelo de gestión cooperativa, destacando diversas líneas como las siguientes:

- Compartir información y crear un clima de comunicación abierta que favorezca la participación de todas las personas en la gestión de la empresa; por ejemplo, mediante reuniones y/o asambleas con todas las personas de la filial.

- Establecer fórmulas que fomenten la participación de los trabajadores en la propiedad de la filial.

-Establecer fórmulas que promuevan la participación de los trabajadores en el reparto de beneficios.

Estas líneas y directrices generales, diseñadas por la tecno-estructura de la Corporación Mondragón, si bien no constituyen normas de obligado cumplimiento, se han traducido en los últimos años en diversas acciones concretas por parte de algunas cooperativas multinacionales dirigidas a promover la 'cooperativización' de las filiales, esto es, la implementación del modelo cooperativo en ellas. Se pueden distinguir dos líneas principales de cooperativización en Mondragón. La primera y más directa consiste en la transformación directa de las filiales capitalistas en cooperativas, y ha sido utilizada exclusivamente en el caso de filiales domésticas. La segunda línea de acción, diseñada principalmente para las filiales extranjeras, se ha basado en la implementación parcial de algunas prácticas de gestión asociadas al modelo cooperativo que caracteriza a las matrices vascas.

\subsubsection{LA COOPERATIVIZACIÓN DE LAS FILIALES DOMÉSTICAS}

La cooperativización de las filiales domésticas se ha llevado a cabo principalmente mediante dos fórmulas. Una implica la creación de una cooperativa mixta ${ }^{2}$, es decir, la filial se convierte en una cooperativa cuya propiedad se reparte, normalmente, entre los propios trabajadores de la filial, la matriz y Mondragón Inversiones S. Coop. La otra implica que,

2 Una cooperativa mixta difiere de una cooperativa convencional fundamentalmente en la estructura de gobierno corporativo. De acuerdo con la Ley Vasca de Cooperativas, las cooperativas mixtas son aquellas que cuentan con accionistas minoritarios, cuyos derechos de voto en la Asamblea General pueden determinarse, exclusiva o preferentemente, sobre la base de sus aportaciones al capital. 
aunque la filial mantiene su forma jurídica, los trabajadores pasan a ser socios de la matriz cooperativa bajo la figura del socio en comisión de servicios ${ }^{3}$, accediendo así a la propiedad, resultados y gestión de la empresa.

Estas experiencias no son nuevas en el grupo Mondragón. Ya en el ańo 2006, alrededor del $15 \%$ del empleo industrial del grupo provenía de esquemas de cooperativización de este tipo (TuLankide, 2006). Sin embargo, los contextos y dinámicas de cooperativización han evolucionado a lo largo de las décadas. En los primeros años, no era posible unirse a Mondragón bajo fórmulas organizativas distintas de la cooperativa. Por lo tanto, algunas empresas capitalistas se incorporaron al grupo transformándose en cooperativas con la ayuda de Caja Laboral y otras cooperativas. En cambio, con el crecimiento de muchas cooperativas de Mondragón a través de la adquisición o creación de filiales capitalistas, se comenzaron también a cooperativizar estas empresas filiales. Algunos de las primeras experiencias desarrolladas por cooperativas multinacionales se llevaron a cabo en Fagor Electrodomésticos. A finales de los 90, la empresa filial vasca Fabrelec S.A. se convirtió en cooperativa (denominada posteriormente Edesa S. Coop.), cuya propiedad se repartió entre los socios trabajadores de la filial, con un 44\%, y la matriz Fagor Electrodomésticos. En 2004, los 250 trabajadores de la filial vasca Geyser Gastech se convirtieron en socios en comisión de servicios de la matriz (Errasti y Mendizabal, 2007).

En los últimos años, también se han llevado a cabo experiencias particularmente notables en este ámbito (para análisis detallados de la cooperativización de filiales domésticas en Mondragón, véase Flecha y Ngai, 2014; Bretos y Errasti, 2016, 2017), destacando los casos de Maier y Fagor Ederlan. En 2006, la mayoría de los 80 trabajadores de la filial vasca Fit Automoción S.A. se convirtieron en socios en comisión de servicios de la empresa matriz Fagor Ederlan; proceso similar al completado recientemente en su otra filial vasca Victorio Luzuriaga Usurbil. Por otro lado, en 2008, Victorio Luzuriaga Tafalla S.A., filial de Fagor Ederlan con sede en Navarra, se transformó en la cooperativa mixta Fagor Ederlan Tafalla S. Coop., pasando algo más de la mitad de sus 700 trabajadores a ostentar la condición de socios cooperativistas. En 2012, la cooperativa Maier llevó a cabo un proceso similar en su filial gallega Maier Ferroplast S.L., donde 150 de los 190 trabajadores de la plantilla aprobaron la transformación de la filial en la cooperativa mixta Maier Ferroplast S. Coop. Nuestra investi-

${ }^{3}$ Los socios en comisión de servicios son aquellos que mantienen un vínculo societario con la cooperativa y prestan su trabajo en una entidad con la que la cooperativa coopera o participa. 
gación sobre estas filiales identificó diversos efectos positivos derivados de su transformación en cooperativas, como la mayor resiliencia de las empresas en los años de crisis económica, la mejora de la estabilidad laboral y de las relaciones entre trabajadores y dirección, la mayor participación de los trabajadores en la empresa y el consecuente aumento de su motivación y compromiso, o la mejora de las condiciones laborales.

No obstante, existen también limitaciones importantes en estos procesos que obstaculizan la genuina implantación de las prácticas y cultura cooperativistas incluso a pesar de la transformación legal de estas empresas en cooperativas. Una limitación fundamental es el acceso restringido de los nuevos socios a la propiedad de las filiales. Por ejemplo, en Fagor Ederlan Tafalla, los trabajadores adquirieron únicamente el $12 \%$ de la empresa, quedando el resto en manos de la matriz Fagor Ederlan y de Mondragón, que conforman mayoría en los órganos de gobierno de la filial cooperativa. En el caso de Maier Ferroplast, el menor tamaño de la filial permitió que los trabajadores adquiriesen el 33\%. Ello se traduce en un cierto descontento entre los trabajadores con la naturaleza y alcance de su participación en la empresa. Por ejemplo, una encuesta interna realizada por Fagor Ederlan Tafalla un año después de la cooperativización de la filial señalaba que el $23 \%$ de los trabajadores de la filial puntuaron su participación en la empresa con un 1 sobre 10. Por otro lado, es evidente la carencia en el compromiso y conocimiento entre los nuevos socios con la cultura y valores cooperativos. Un año después de la cooperativización, sólo un 7\% de los trabajadores de Fagor Ederlan Tafalla afirmaron conocer el contenido y significado de los principios y valores de la Experiencia Cooperativa de Mondragón, reconociendo que la mayoría se convirtieron en socios por la mayor estabilidad laboral que ofrece la fórmula cooperativa. Asimismo, los trabajadores que se mantuvieron como asalariados tras los procesos de cooperativización quedaron excluidos de los espacios democráticos y participativos de las filiales (Bretos y Errasti, 2016, 2017).

\subsubsection{LA COOPERATIVIZACIÓN DE LAS FILIALES EXTRANJERAS}

Como señalábamos anteriormente, la cooperativización de las filiales extranjeras se ha basado en la implantación parcial de ciertas prácticas de gestión vinculadas al modelo cooperativo. Algunos trabajos que han analizado este tipo de iniciativas señalan que las cooperativas multinacionales de Mondragón han sido capaces de mantener los valores y prácticas cooperativistas durante la expansión internacional y de extender el modelo cooperativo a las filiales extranjeras (Luzarraga, 2008; Luzarraga e Irizar, 2012; Flecha y Ngai, 2014; Santos-Pitanga, 2015). El argumento de estos autores 
se basa fundamentalmente en la introducción de determinadas prácticas en las filiales, como la organización en equipos auto-gestionados, la formación de los trabajadores en aspectos técnicos, o la mayor comunicación entre los empleados y la dirección.

En cambio, nuestra investigación sobre diversas filiales extranjeras del grupo Mondragón arrojó unos resultados notablemente diferentes (Errasti, 2015; Errasti et al., 2016; Bretos y Errasti, 2017; Bretos et al., 2017). En términos generales, las cooperativas multinacionales de Mondragón han introducido tres tipos de prácticas de manera similar en todas las filiales extranjeras. En primer lugar, todas las filiales extranjeras operan bajo el mismo modelo de Gestión de la Calidad Total y técnicas de producción ajustada, con estándares medioambientales, de seguridad y de calidad equivalentes, por lo que la organización del trabajo en estas filiales es bastante similar a la existente en las matrices cooperativas. En segundo lugar, las cooperativas de Mondragón han implementado mecanismos de participación directa para los trabajadores. Por ejemplo, todas las filiales han introducido el modelo de las mini-compañías, algún tipo de sistema de sugerencias del empleado, y el establecimiento de reuniones de periódicas entre gerencia y trabajadores que fomentan el intercambio de información sobre la producción y otros aspectos técnicos. De esta forma, la participación de los trabajadores en el área de trabajo es notable en las filiales extranjeras. En tercer lugar, todas las filiales extranjeras han introducido sistemas de retribución variable. En el caso de los directivos, las recompensas se establecen conforme a objetivos establecidos periódicamente. En el caso de los trabajadores de planta, las recompensas están vinculadas a la productividad y a la calidad de la producción.

Por otra parte, en lo relativo a otras prácticas de gestión asociadas al modelo cooperativo (como la equidad salarial, la estabilidad laboral, la promoción interna, y la formación continua de los trabajadores), se observan diferencias sensibles entre las filiales localizadas en distintos países. Por ejemplo, es evidente que en las caso de las filiales chinas apenas existe rastro del modelo cooperativo (Errasti, 2015; Bretos et al., 2017). En cambio, en otras filiales se ha logrado avanzar significativamente en la introducción de este tipo de prácticas. Un ejemplo es Fagor Ederlan Brasileira, la filial brasileña de Fagor Ederlan. Esta filial ha introducido un balance social con indicadores de desempeńo económico, social y ambiental que facilitan la comparación de las características de la empresa matriz con las de la filial. Entre otros aspectos, se han reducido las diferencias en las escalas salariales de la empresa, se han promovido las oportunidades de formación y de promoción interna para los trabajadores, las condiciones laborales se revisan anualmente en colaboración con los sindicatos para mantenerlas en niveles similares o superiores a los del entorno local, y se han añadido beneficios 
sociales para los trabajadores que incluyen, entre otros, seguros de salud, cupones de comidas y el transporte a la planta. Todo ello ha fomentado la estabilidad laboral y el bienestar de los trabajadores (Bretos y Errasti, 2017; Bretos et al., 2017). Igualmente, antes del impacto de la crisis económica, Fagor Electrodomésticos también logró avances similares en su planta localizada en Polonia (Errasti et al., 2016).

Sin embargo, aunque el modelo cooperativo se ha afianzado en mayor media en algunas filiales, es evidente que ninguna de ellas ha sido transformada en cooperativa ni ha introducido de manera consistente el conjunto de prácticas asociadas al modelo cooperativo, en particular, en lo concerniente a la participación de los trabajadores en la propiedad, reparto de beneficios y gestión general de la empresa; aspectos centrales de la fórmula cooperativa. Nuestra investigación identificó diversos factores que dificultan la genuina cooperativización de las filiales extranjeras. Por un lado, existen barreras de carácter cultural e institucional. Por ejemplo, en diversos países donde se localizan las cooperativas de Mondragón no existe una legislación que ampare jurídicamente a la fórmula de la cooperativa de trabajo, como es el caso de China, por ejemplo. Evidentemente, ello dificulta la posible transformación de estas filiales en cooperativas. Además, muchos de estos países tampoco cuentan con una tradición cooperativa como la existente en el País Vasco, y los empleados de están filiales no están acostumbrados a trabajar en una cultura laboral de cooperación. Ello implica en definitiva la dificultad de introducir en estas filiales prácticas de gestión basadas en la participación de los trabajadores y la toma de decisiones colectiva y democrática.

Por otro lado, si bien estos factores institucionales son relevantes, nuestra investigación identificó otras barreras, vinculadas con las relaciones de poder matriz-filial y con los intereses propios en la matriz, que parecen obstaculizar la cooperativización de las filiales extranjeras de manera todavía más crítica. Un aspecto clave es la percepción entre los socios cooperativistas vascos de que los trabajadores en las plantas extranjeras no desarrollarán un compromiso tan sólido como el suyo con la empresa y con la cultura cooperativa, lo que genera en última instancia cierta desconfianza sobre el éxito de un hipotético proyecto de cooperativización en una planta extranjera. Asimismo, los directivos y socios trabajadores de las matrices cooperativas consideran que la mayor participación de los trabajadores y autonomía de las filiales extranjeras podría ser perjudicial para el control de la matriz sobre el conjunto del grupo empresarial, percibiendo por tanto que la cooperativización de plantas extranjeras podrían poner en riesgo la propia viabilidad de la cooperativa y los puestos de trabajo de los socios cooperativistas vascos. 


\section{Conclusiones}

Algunos trabajos recientes han desafiado la visión determinista y monolítica de la teoría de la degeneración demostrando que las cooperativas son capaces de lidiar con las presiones isomorfas institucionales - las cuales empujan a estas organizaciones a adoptar formas organizacionales y prioridades similares a la empresa capitalista- y desarrollar estrategias de regeneración (por ejemplo, Ng y Ng, 2009; Storey et al., 2014; Narvaiza et al., 2017; Jaumier, 2017). Este estudio complementa estas contribuciones sobre la regeneración cooperativa mediante el análisis de las dinámicas degenerativas y regenerativas que tienen lugar en las cooperativas multinacionales, un ámbito donde nuestro conocimiento es extremadamente limitado todavía.

Como evidencia nuestra investigación, las cooperativas y otras organizaciones de la economía social han estado expuestas históricamente a presiones degenerativas. El capitalismo global y la transformación de algunas cooperativas de Mondragón en organizaciones multinacionales no han hecho sino acentuar las tensiones ya existentes entre los principios cooperativos y el éxito empresarial en un entorno capitalista, afectando de esta forma a la gobernanza democrática y a los sistemas participativos en estas grandes cooperativas orientadas al mercado. Estas tensiones están alimentadas por varias dinámicas, como el predominio del control gerencial a expensas de la participación de los trabajadores, la reconfiguración de los valores y prácticas cooperativas de acuerdo con las prioridades gerenciales de eficiencia y competitividad, y la anexión de filiales capitalistas donde los derechos y beneficios asociados al modelo cooperativo, como la estabilidad laboral y la participación en las decisiones corporativas, están restringidos para los trabajadores.

No obstante, nuestro estudio también pone de manifiesto cómo estas cooperativas multinacionales pueden diseñar e implementar diversas estrategias de regeneración orientadas a revitalizar los valores y prácticas cooperativistas, por ejemplo, mediante la recuperación e institucionalización de la educación cooperativa o mediante la profundización de la participación de los trabajadores de base y la dinamización de distintos espacios democráticos para fomentar la involucración y comunicación de todas las personas de la organización. Más allá de estas cuestiones, que atañen fundamentalmente a las cooperativas matrices, este trabajo ha hecho especial hincapié en las iniciativas de cooperativización desarrolladas en los últimos años en las filiales capitalistas, tanto domesticas como internacionales, que tienen por objeto extender el modelo cooperativo en las mismas. Si bien estas iniciativas de cooperativización no están exentas de desafíos y limitaciones, su potencial transformador es evidente. Previsiblemente, este tipo de estrategias de regeneración seguirán siendo fundamentales en los próximos años, 
teniendo en cuenta que cada vez más cooperativas se están expandiendo internacionalmente a través de la adquisición y creación de empresas capitalistas (Bretos y Marcuello, 2017). Asimismo, este escenario abre importantes líneas de investigación futuras. Por ejemplo, una línea fundamental consiste en estudiar cómo la implementación del modelo cooperativo en estas filiales influye no sólo en la productividad y desempeño organizacional de la empresa, sino en el bienestar de los trabajadores y en otros aspectos como el desarrollo local sostenible de los territorios donde se sitúan dichas filiales. Otra cuestión esencial, en la que se están comenzando a dar los primeros pasos (Bretos et al., 2017), reside en comprender los desafíos que plantea la gobernanza en las cooperativas multinacionales y cómo éstas pueden diseñar políticas internacionales de gestión de recursos humanos que equilibren la orientación cooperativa basada en el bienestar del trabajador con la orientación económica necesaria para competir en los mercados internacionales.

Finalmente, esta investigación también tiene importantes implicaciones para el desarrollo de la teoría organizacional de las cooperativas. A diferencia de los defensores de la tesis degenerativa, que asumen que las tensiones y limitaciones existentes en las cooperativas para equilibrar su dimensión económica y social implicarán ineludiblemente el fracaso comercial o democrático de la organización, este estudio sugiere que la mejor manera de abordar y comprender las tensiones existentes en cooperativas que compiten en una economía de mercado es mediante un 'enfoque paradójico' (Hernández, 2006; Ashforth y Reingen, 2014). Desde esta perspectiva, las tensiones y paradojas son elementos inherentes en la supervivencia de organizaciones híbridas - organizaciones que combinan una lógica institucional de carácter empresarial y social simultáneamente- como son las cooperativas. El desafío para las cooperativas se encuentra, por tanto, en encontrar un equilibrio dinámico orientado a desencadenar cambios organizacionales positivos dentro de una lucha perenne entre la resistencia de los trabajadores y el control gerencial (Courpasson et al., 2012). En nuestra opinión, el día que ya no encontremos tensiones, paradojas y contradicciones en las cooperativas será porque han dejado de ser alternativas y han perdido su potencial transformador. Mientras tanto, las cooperativas tendrán que sobrevivir con, a través, y más allá de las tensiones.

\section{Referencias bibliográficas}

Altuna, L., Loyola, A. y Pagalday, E. (2013): Mondragón: The Dilemmas of a Mature Cooperativism. En: C. Piñeiro-Harnecker (Ed.), Cooperatives and Socialism: A View from Cuba, pp. 167-188. New York: Palgrave Macmillan. 
Arando, S., Gago, M., Jones, D.C. y Kato, T. (2015): Efficiency in employeeowned enterprises: An econometric case study of Mondragon. ILR Review 68(2): 398-425.

Ashforth, B.E. y Reingen, P.H. (2014): Functions of Dysfunction Managing the Dynamics of an Organizational Duality in a Natural Food Cooperative. Administrative Science Quarterly 59(3): 474-516.

Atzeni, M. (ed.) (2012): Alternative Work Organizations. London: Palgrave Macmillan.

Azkarraga, J., Cheney, G. y Udaondo, A. (2012): Workers Participation in a Globalized Market: Reflections on and from Mondragon. En: M. Atzeni (ed.), Alternative Work Organisations, pp. 76-102. New York: Palgrave Macmillan.

Basterretxea, I. y Albizu, E. (2011): Management Training as a Source of Perceived Competitive Advantage: The Mondragon Cooperative Group Case. Economic and Industrial Democracy 32(2): 199-222.

Batstone, E. (1983): Organization and Orientation: A Life Cycle Model of French Cooperatives. Economic and Industrial Democracy 4(2): 139-161.

Ben-ner, A. (1984): On the Stability of the Cooperative Type of Organization. Journal of Comparative Economics 8(3): 247-260.

Bretos, I. y Errasti, A. (2016) Dinámicas de regeneración en las cooperativas multinacionales de Mondragón: la reproducción del modelo cooperativo en las filiales capitalistas. CIRIEC-España, Revista de Economía Pública, Social y Cooperativa $86: 5-34$.

Bretos, I. y Morandeira, J. (2016): La economía social ante la actual crisis económica en la Comunidad Autónoma del País Vasco. REVESCO, Revista de Estudios Cooperativos 122: 7-33.

Bretos, I. y Errasti, A. (2017): Challenges and Opportunities for the Regeneration of Multinational Worker Cooperatives: Lessons from the Mondragon Corporation-A Case Study of the Fagor Ederlan Group. Organization 24(2): 154173.

Bretos, I. y Marcuello, C. (2017): Revisiting Globalization Challenges and Opportunities in the Development of Cooperatives. Annals of Public and Cooperative Economics 88(1): 47-73.

Bretos, I., Errasti, A. y Marcuello, C. (2017): Ownership, governance, and the diffusion of HRM practices in multinational worker cooperatives: Case-study evidence from the Mondragon group. Human Resource Management Journal DOI: $10.1111 / 1748-8583.12165$.

Cathcart, A. (2013): Directing democracy: Competing interests and contested terrain in the John Lewis Partnership. Journal of Industrial Relations 55(4): 601-620.

Cathcart, A. (2014): Paradoxes of Participation: Non-Union Workplace Partnership in John Lewis. International Journal of Human Resource Management 25(6): 762-780.

Cheney, G. (2002): Values at Work: Employee Participation Meets Market Pressure at Mondragon. Ithaca, NY: Cornell University Press. 
Cheney, G. (2005): Democracy at Work within the Market: Reconsidering the Potential. En: V. Smith (ed.), Worker Participation: Current Research and Future Trends, pp. 179-203. Amsterdam: Emerald Group Publishing Limited.

Cooke, P., Uraga, M.G. y Etxebarria, G. (1997): Regional innovation systems: institutional and organisational dimensions. Research Policy 26: 475-91.

Cornforth, C. (1995): Patterns of cooperative management: Beyond the degeneration thesis. Economic and Industrial Democracy 16(4): 487-523.

Courpasson, D., Dany, F., y Clegg, S. (2012): Resisters at work: Generating productive resistance in the workplace. Organization Science 23: 801-819.

Elortza, E., Alzola, I. y López, U. (2012): La gestión de la crisis en la Corporación Mondragón. Ekonomiaz 79(1): 58-81.

Errasti, A. (2015): Mondragon's Chinese subsidiaries: Coopitalist multinationals in practice. Economic and Industrial Democracy 36: 479-499.

Errasti, A. y Mendizabal, A. (2007): The impact of globalization and relocation strategies in large co-operatives: The case of the Mondragon Co-operative Fagor Electrodomésticos S.Coop. Advances in the Economic Analysis of Participatory and Labor-managed Firms 10: 265-287.

Errasti, A., Bretos, I. y Etxezarreta, E. (2016): What do Mondragon Coopitalist Multinationals Look Like? The Rise and Fall of Fagor Electrodomésticos S. Coop. and its European Subsidiaries. Annals of Public and Cooperative Economics 87: 433-456.

Errasti, A., Bretos, I. y Nunez, A. (2017): The Viability of Cooperatives: The Fall of the Mondragon Cooperative Fagor. Review of Radical Political Economics 49(2): 181-197.

Flecha, R. y Ngai, P. (2014): The challenge for Mondragon: Searching for the cooperative values in times of internationalization. Organization 21(5): 666-682.

Greenwood, D. y Gonzalez, J.L. (1990): Culturas de Fagor: Estudio antropológico de las cooperativas de Mondragón. Donostia: Editorial Txertoa.

Harvey, D. (2005): A Brief History of Neoliberalism. Oxford: Oxford University Press.

Heras, I. (2014): The Ties that Bind? Exploring the Basic principles of WorkerOwned Organizations in Practice. Organization 21(5): 645-665.

Heras, I. y Basterretxea, I. (2016): Do co-ops speak the managerial lingua franca? An analysis of the managerial discourse of Mondragon cooperatives. Journal of Co-operative Organization and Management 4(1): 13-21.

Hernandez, S. (2006): Striving for control: Democracy and oligarchy at a Mexican cooperative. Economic and Industrial Democracy 27(1): 105-135.

Irizar, I. (2005): Empresa Cooperativa y Liderazgo. Oñati: Mondragon Unibertsitatea.

Jaumier S. (2017): Preventing chiefs from being chiefs: An ethnography of a cooperative sheet-metal Factory. Organization 24(2): 218-239.

Kasmir, S. (1996): The Myth of Mondragon, Cooperatives, Politics and WorkingClass Life in a Basque Town. New York: SUNY Press. 
Kasmir, S. (2016): The Mondragon Cooperatives and Global Capitalism: A Critical Analysis. New Labor Forum 25(1): 52-59.

Luxemburg, R. (1900): Reform or Revolution. London: Militant Publications.

Mandel, E. (1975): Self-Management Dangers and Possibilities. International 2(4): 3-9.

Luzarraga, J.M. (2008): Mondragon Multi-Localisation Strategy: Innovating a Human Centred Globalisation. PhD Thesis, Mondragon Unibertsitatea, Spain.

Luzarraga, J.M. e Irizar, I. (2012): La Estrategia de Multilocalización Internacional de la Corporación Mondragón. Ekonomiaz 79: 114-145.

Marx, K. (1967): Capital: A Critique of Political Economy, vol. 1. New York: International Publishers.

McMurtry, J. y Reed, D. (2009): Co-operatives in a Global Economy: The Challenges of Co-operation across Borders. Newcastle, UK: Cambridge Scholars Publishing.

Meister, A. (1974): La participation dans les associations. Paris: Editions Ouvrières.

Meister, A. (1984): Participation, Associations, Development and Change. New Brunswick, NJ: Transaction Books.

Miyazaki, H. (1984): On Success and Dissolution of the Labour-Managed Firm in the Capitalist Economy. Journal of Political Economy 92(5): 909-931.

Molina, F. y Miguez, A. (2008): The origins of Mondragon: Catholic co-operativism and social movement in a Basque valley (1941-59). Social History 33(3): 284-298.

Mondragon (2013): Corporate Management Model. Mondragón: Mondragon Corporation.

Mondragon (2015): A review of the key milestones in the co-operative group's history. Eskoriatza: Mondragon Corporation.

Mondragon (2016): 2015 Annual Report. Eskoriatza: Mondragon Corporation.

Narvaiza, L., Aragon-Amonarriz, C., Iturrioz-Landart, C., Bayle-Cordier, J. y Stervinou, S. (2017): Cooperative Dynamics during the Financial Crisis: Evidence from Basque and Breton Case Studies. Nonprofit and Voluntary Sector Quarterly 46(3): 505-524.

$\mathrm{Ng}$, C.W. y Ng, E. (2009): Balancing the democracy dilemmas: Experiences of three women workers' cooperatives in Hong Kong. Economic and Industrial Democracy 30 (2): 182-206.

Paranque, B. y Willmott, H. (2014): Cooperatives—saviours or gravediggers of capitalism? Critical performativity and the John Lewis Partnership. Organization 21(5): 604-625.

Parker, M., Cheney, G., Fournier, V. y Land, C. (eds.) (2014): The Routledge Companion to Alternative Organization. London: Routledge.

Rosner, M. (1984): A Search for 'Coping Strategies' or Forecasts of Cooperative Degeneration? Economic and Industrial Democracy 5(3): 391-399.

Russell, R. (1984): The role of culture and ethnicity in the degeneration of democratic firms. Economic and Industerial Democracy 5(1): 73-96. 
Santos-Pitanga, T. (2015): La institucionalización de la solidaridad: El caso Mondragón. PhD thesis, Universitat de Barcelona, Spain.

Storey, J., Basterretxea, I. y Salaman, G. (2014): Managing and resisting 'degeneration' in employee-owned businesses: A comparative study of two large retailers in Spain and the United Kingdom. Organization 21(5): 626-644.

Stryjan, Y. (1994): Understanding cooperatives: The reproduction perspective. Annals of Public and Cooperative Economics 65(1): 59-80.

Taylor, P.L. (1994): The Rhetorical Construction of Efficiency: Restructuring and Industrial Democracy in Mondragon, Spain. Sociological Forum 9(3): 459-89.

TuLankide (2006): De S.A. hacia S. Coop. TuLankide 509: 3-4.

Varman, R. y Chakrabarti, M. (2004): Contradictions of Democracy in a Workers' Cooperative. Organization Studies 25(2): 183-208.

Webb, T. y Cheney, G. (2014). Worker-owned-and-governed co-operatives and the wider co-operative movement. En: M. Parker, G. Cheney, V. Fournier and C. Land (eds), The Routledge Companion to Alternative Organization, pp. 64-88. London: Routledge.

Webb, S. y Webb, B. (1914): The Cooperative Movement. London: Longmans.

Webb, S. y Webb, B. (1920): A constitution for the socialist commonwealth of Great Britain. London: Longmans.

Whyte, W.F. y Whyte, K.K. (1991): Making Mondragon: The Growth and Dynamics of the Worker Cooperative Complex. Ithaca, NY: ILR Press. 